\title{
Al, COVID-19 and the long haul
}

\author{
It has been a little over a year since a worldwide COVID-19 pandemic was declared. Science has moved fast to fight \\ the virus but preparations need to be underway for fighting future outbreaks.
}

sp he novel coronavirus SARS-CoV-2 spread fast and wide, causing much damage to human health and wellbeing across the globe. Fortunately, science also moved fast. Several working vaccines against COVID-19 have been developed within a year at unprecedented speed, and inoculation programs, together with improving patient treatments and regular testing, point to a way out of this pandemic. There are no guarantees we'll be able to return to a normal life soon though and the virus can still test us with new local outbreaks and dangerous mutations. A continuing global collaboration to keep track of the virus, and renewed efforts to ensure that all of the world's population is signed up to vaccine programs and can benefit from accumulating knowledge on the disease, seem essential. The risks posed by coronavirus will be with us for a long time and preparations for fighting outbreaks in the near future need to be underway.

Researchers from many fields are responding to this call. In a workshop last summer organized by the National Academy of Engineers (NAE) and Computing Community Consortium (CCC), scientists, frontline health workers, and public health and safety experts came together, virtually of course, to discuss the role of robotics in infectious diseases. Robotics can be of crucial help to look after patients while minimizing spread of infection and protecting healthcare staff. However, to play a significant role in future crises, developments should be strategically planned now and in collaboration with healthcare workers and hospitals. In a Comment in this issue, Hao Su et al. describe a vision for robots that can be deployed for physical human-robot interaction applications in healthcare. Reviewing the state of the art in robotics, they conclude that advances are required in robot sensing, dexterous manipulation and autonomy capabilities.

Another focus area is the development of machine learning models to help in the diagnosis and prognosis of COVID-19. However, while model development can be very fast, frontline healthcare environments cannot benefit from these advances without more attention on the longer process of rigorous validation and adaptation to local healthcare. An Analysis in this issue by Michael Roberts et al. reviews all preprints and papers published since the outbreak of COVID-19 up to 3 October 2020 on detection and prognostication based on clinical chest X-ray and computed tomography (CT) imaging. The hard lesson found by the authors is that none of the developed models, at least so far, can be of potential clinical use due to methodological flaws and high or unclear risk of bias in the datasets used. The authors offer recommendations for improving the quality of models, such as the use of a well-curated external validation set. They also encourage researchers to assess their models against established quality frameworks, in particular the recently developed Checklist for Artificial Intelligence in Medical Imaging (CLAIM).

A different focus area for the fight against COVID-19 where machine learning holds promise is in drug repurposing and drug testing. Developing new drugs is costly and time-consuming, and given the urgent need for treatments, efforts are directed towards exploring existing drugs, already approved or under investigation, to treat COVID-19. AI approaches are well suited for trawling through large databases and combining information from different sources. In an Article in this issue, Ping Zhang and colleagues report a graph neural network approach for drug repurposing that combines gene-expression data and drug interaction databases. The authors fortuitously completed the project around May 2020 and could immediately test it on COVID-19, for which gene expression data were available. They identified 15 existing drugs that could be used for treating COVID-19. Among those are several drugs that are already approved for clinical use in the treatment of other diseases.

Ultimately, deciding which drugs to develop and trial will be made by clinicians. But AI can help identify candidate drugs and treatments, and can even take some of the burden of clinical trials, which is especially useful for a new disease such as COVID-19 that is not yet well understood and has complex biological and clinical symptoms. Randomized clinical trials on repurposed drugs can be simulated by data-driven methods and computer models by processing existing data such as medical health records and insurance claims retrospectively. Such real-world data have many confounding factors, but with sufficient dataset size, useful information can be teased out by AI, which is invaluable in the fight against a fast-developing infectious disease.

We're in for the long haul in the battle to control COVID-19. But some optimism is warranted, with scientific research well placed to prepare for future outbreaks.

Published online: 18 March 2021 https://doi.org/10.1038/s42256-021-00328-9 